\title{
Behavioral and anatomical consequences of unilateral fornix lesions and the administration of nimodipine
}

\author{
Anne M. Danks*, A.B. Oestreicher, B.M. Spruijt, W.H. Gispen and Robert L. Isaacson \\ State University of New York, Binghamton, NY 13902-6000 (U.S.A.)
}

(Accepted 30 April 1991)

Key words: Fornix lesion; Septal area; Nimodipine; Water maze

\begin{abstract}
Male Wistar rats subjected to unilateral fimbria-fornix transection by mechanical knife cut or to sham operations were tested in a water maze and in an open field. Half the animals in each group were treated with either $0.06 \mathrm{mg} / \mathrm{kg}$ nimodipine or vehicle, administered i.p. for 7 days, beginning the day of surgery. Animals were sacrificed and brains were processed for acetylcholine esterase (AChE) histochemistry. In the water maze, lesioned rats showed a significant impairment relative to the sham-operated animals. Nimodipine treatment did not improve performance. There were no differences among the groups in the observed frequencies of the open field behaviors of locomotion, hole-poke, rearing and grooming. A significant reduction of AChE-positive cell bodies was found in the medial septal region on the side of the lesion. There were no differences in water maze performance among groups of rats treated with $0.0,0.5,1.0$, or $5.0 \mathrm{mg} / \mathrm{kg} \mathrm{nimodipine} \mathrm{for} 7 \mathrm{days}$, beginning the day of fimbria-fornix transection, in an attempt to determine any dose-dependent effect of the drug.
\end{abstract}

Until recently, no reliable behavioral effects had been demonstrated as a consequence of unilateral lesions of the fimbria-fornix. However, in 1988 a robust behavioral deficit was found following unilateral lesions in a rather complex water maze ${ }^{3}$. Subsequently, similar unilateral lesions have been shown to produce a performance deficit in the Morris water maze and in a passive avoidance $\operatorname{task}^{16,20}$.

In addition to behavioral deficits, lesions of the fimbria-fornix produce marked depletions in the cholinergic enzymes acetylcholine esterase (AChE) and choline acetyltransferase (ChAT) $)^{2,5,6,7,10}$. Disruption of the axons in the fimbria-fornix causes retrograde changes of the cholinergic cells in the medial septal region ${ }^{1,4,15}$.

It has been suggested that at least certain injuries to neurons cause excessive $\mathrm{Ca}^{2+}$ influx that may ultimately prove toxic to the cells ${ }^{18,19}$, although the exact mechanism of this toxicity remains unclear. Accordingly, a reduction in this intracellular accumulation of calcium following injury may act to reduce subsequent damage or dysfunction.

Among several classes of compounds being tested for their ability to block voltage-sensitive $\mathrm{Ca}^{2+}$ channels are certain dihydropyridines (DHP). The $\mathrm{Ca}^{2+}$ channel antagonist nimodipine, a 1,4-dihydropyridine derivative, has been shown to facilitate recovery of sensorimotor function after crush lesion of the sciatic nerve in rats ${ }^{21}$, as well as behavioral recovery of animals subjected to hypoxia $^{13,14}$. The administration of nimodipine after lesion of the fimbria-fornix has been reported to cause a sparing of cells expressing AChE in the medial septal area ${ }^{11}$. If nimodipine is able to afford a protective effect on a cellular level in this region, then an accompanying attenuation of the behavioral impairments produced by this lesion might also be expected. This study was undertaken to find a reliable behavioral indicator of unilateral fimbria-fornix transections, and to determine any possible beneficial effects of systemic administration of nimodipine.

Group housed male Wistar rats (TNO, Zeist, Netherlands), weighing $220-240 \mathrm{~g}$, were used in all experiments. Food and water were available ad libitum. The unilateral fimbria-fornix transections or sham operations were performed with the animals given $0.1 \mathrm{ml} / 100 \mathrm{~g}$ body weight Hypnorm (Philips Duphar, Netherlands). Lesions were made with a modified stainless steel scalpel blade from $\mathrm{L}=1.5-5.5 \mathrm{~mm}, \mathrm{~A}=6.2 \mathrm{~mm}, \mathrm{D}=8.0 \mathrm{~mm}$ from top of skull ${ }^{10}$. All lesions were made in the left hemisphere. Sham lesions were produced by lowering the knife through the cortex to $\mathrm{D}=3.0 \mathrm{~mm}$. Rats were

\footnotetext{
* This research was conducted while the senior author (A.M.D.) was a visiting scholar at the Division of Neurobiology, Institute of Molecular Biology, University of Utrecht, Utrecht, The Netherlands. An abstract of this research was published in Soc. Neurosci. Abstr., 14 (1988) 1246.

Correspondence: R.L. Isaacson, State University of New York, P.O. Box 6000, Binghamton, NY 13902-6000, U.S.A.
} 
injected i.p. with either $0.06 \mathrm{mg}$ nimodipine (Bayer, A.G., F.R.G.) in a volume of $0.2 \mathrm{ml}$, or an equal volume of vehicle alone, for 7 consecutive days, beginning the day of surgery. The vehicle was a mixture of PEG 400 (polyethyleneglycol) diluted 1:1 with distilled water.

Rats were tested in a water maze $(75 \mathrm{~cm} \times 120 \mathrm{~cm}$, Fig. 1) for 3 consecutive days beginning on postoperative day 11 for the '14-day' recovery group or on postoperative day 25 for the '28-day' recovery group. Water temperature in the maze was $20^{\circ} \mathrm{C}$. Prior to the first trial on the first day of testing, animals were placed at the bottom of the escape grid and allowed to climb it, remaining at the top of the grid for approximately $5 \mathrm{~s}$. The amount of time taken to escape the maze, up to a maximum of $210 \mathrm{~s}$, was recorded on each trial. If an animal failed to escape the maze within $210 \mathrm{~s}$, it was grasped about the middle by the experimenter and guided correctly through the remainder of the maze. The number of 'errors' made on each trial was also recorded. Errors were defined as entering a blind alley or reversing direction in the maze. The intertrial interval was approximately $1 \mathrm{~h}$.

The day following behavioral testing animals were sacrificed by an overdose of Hypnorm and perfused intracardially with $0.9 \%$ sodium phosphate buffered saline (PBS) followed by $2 \%$ paraformaldehyde, $0.5 \%$ glutaraldehyde in PBS.

In a second set of rats, animals underwent the same surgical and drug treatments as described above, but were tested in a $100 \mathrm{~cm} \times 100 \mathrm{~cm} \times 10 \mathrm{~cm}$ open field 13 days after surgery. The open field was divided into a $5 \times$ 5 matrix with holes of $3 \mathrm{~cm}$ diameter located at the intersections of the lines on the floor. Frequency scores of locomotion, hole-poke, rearing, and grooming were obtained for a 10 min period, as described previously ${ }^{17}$. Animals were sacrificed and perfused on postoperative day 14 , as described above.

In a third set of animals, fimbria-fornix transections

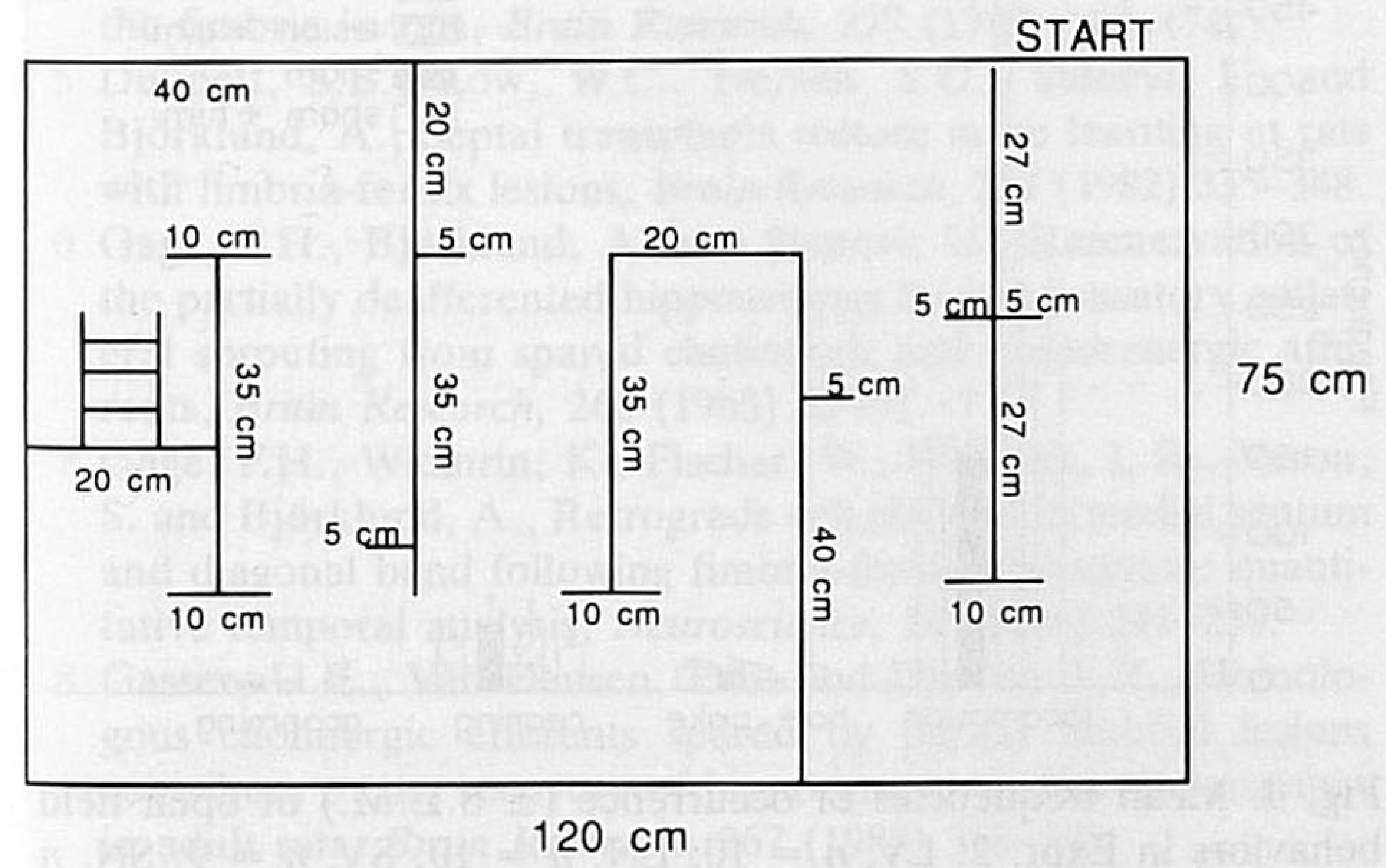

Fig. 1. Schematic drawing of the water maze used with dimensions. were made and the rats injected i.p. with $0.5 \mathrm{mg} / \mathrm{kg}, 1.0$ $\mathrm{mg} / \mathrm{kg}$, or $5.0 \mathrm{mg} / \mathrm{kg}$ nimodipine, or the vehicle. On post-operative days $11-13$, animals were tested in the water maze described above, and sacrificed on postoperative day 14 .

Prior to the production of frozen sections, the brains were cryoprotected with sucrose. The sections were made in the horizontal plane at $24 \mu \mathrm{m}$ and transferred to poly-L-lysine coated microscope slides. Sections were stained for AChE according to the method of Karnovsky and Roots, Hedreen modification ${ }^{9,12}$. Control slides were processed without acetylthiocholine iodide or with isoOMPA $\left(10^{-5} \mathrm{M}\right)$ added as an inhibitor of non-specific esterases.

Absence of staining in the hippocampus ipsilateral to the lesion and microscopic examination of Cresyl violet stained horizontal sections through the level of the fornix were used as indices of lesion completeness. Data from those animals whose histological sections indicated an incomplete lesion of the fornix were discarded from the study.

Cells positively stained for $\mathrm{AChE}$ on the left and right sides of the medial septal region were counted through a microscope $(100 \times$ magnification) by two independent observers. The area examined was bounded ventrally at the level of the anterior commissure and dorsally at the level where the cingulum becomes discontinuous with the genu of the corpus callosum. Four to eight sections were counted for each animal. The side contralateral to the lesion was used as the 'control'. The results are presented as percentage of remaining AChE-positive cells on the lesioned, relative to the contralateral, side (ipsilateral/ contralateral $\times 100$ ).

Water maze data were analyzed using a $2 \times 2 \times 10$ ANOVA (surgery $\times$ drug $\times$ trial, with trial as a within-subject repeated measure). Follow-up comparisons were made using Tukey (hsd) analysis.

The amount of time taken to escape the maze (latency) is shown in Fig. 2 (upper: 14-day recovery group; lower: 28-day recovery group). At both post-surgical periods, the lesioned animals showed a marked impairment in acquisition of the escape route [14-day groups: $F_{1,34}=$ 11.446, $P<0.002 ; 28$-day groups: $F_{1,47}=5.189, P<$ $0.03]$. Nimodipine treatment itself did not affect water maze performance at either postoperative period.

Analysis of the error data closely paralleled the latency data, and are not shown. With both the 14- and 28-day recovery groups there were significant effects of surgery and trial, with no other significant main effects or interactions. Essentially, as the animals' latencies to escape the maze decreased, the number of errors made also decreased.

The typical extent of the fimbria-fornix transection is 


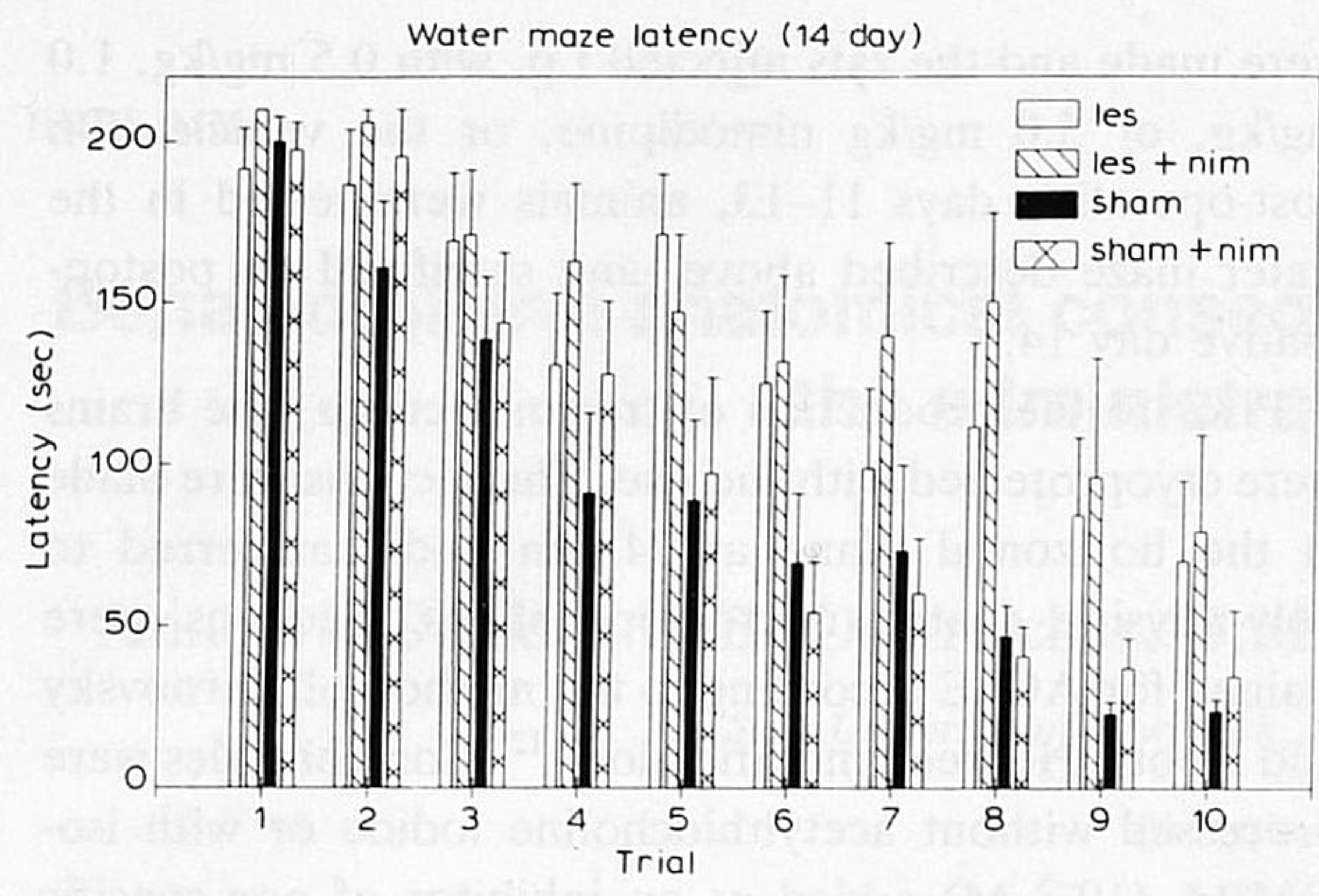

Water maze latency (28 day)

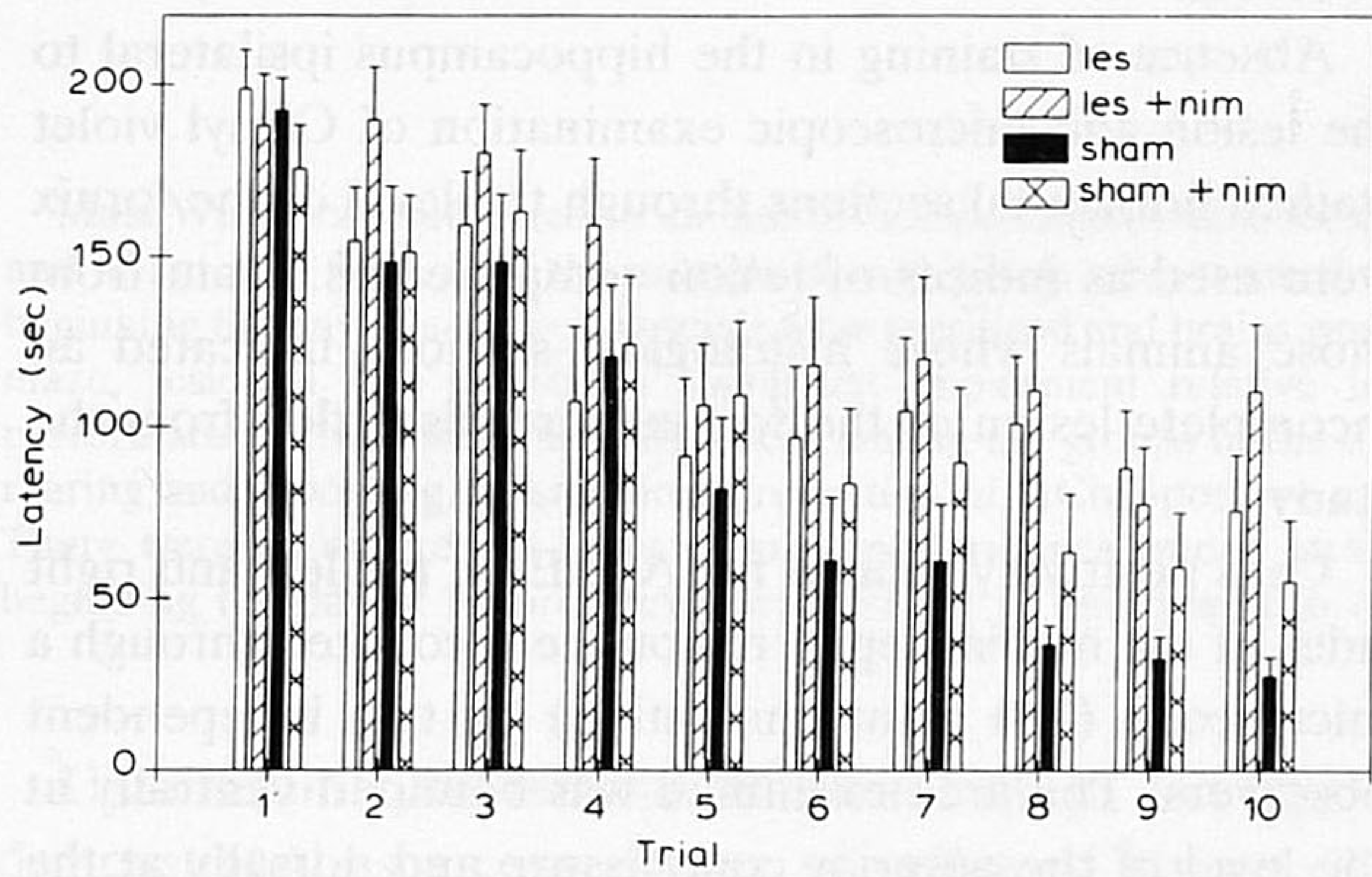

Fig. 2. Upper: mean times ( \pm S.E.M.) taken to escape the water maze for animals allowed 14-day recovery. LV, $n=10 ; \mathrm{LN}, n=10$; SV, $n=9 ; \mathrm{SN}, n=9$. Lower: mean times ( \pm S.E.M.) taken to escape the water maze for animals allowed 28-day recovery. LV, $n=$ 12 ; LN, $n=12$; SV, $n=13$; SN, $n=14$.

shown in a Cresyl violet stained section in Fig. 3. The percentage of AChE-positive cells in the medial septal region remaining on the lesioned side of the brain is reported in Table I. Since the data for the 14- and 28-day recovery periods did not significantly differ, the data

\section{TABLE I}

Percent of AChE-positive cells remaining in medial septum on the side of the lesion relative to the unlesioned side

$\mathrm{LV}, n=4 ; \mathrm{LN}, n=7 ; \mathrm{SN}, n=2 ; \mathrm{SN}, n=5$. Groups refer to whether or not the rats had fornix lesions. 'Vehicle' and 'Nimodipine' refer to whether the animals in the group were given nimodipine or the vehicle on the day of surgery and for several days afterward.

\begin{tabular}{lcc}
\hline Group & Vehicle & Nimodipine \\
\hline Lesion & & \\
Mean & 59.8 & 54.4 \\
S.E.M. & 4.3 & 3.7 \\
& & \\
Sham & & \\
Mean & 84.3 & 93.0 \\
S.E.M. & 6.2 & 4.5 \\
\hline
\end{tabular}

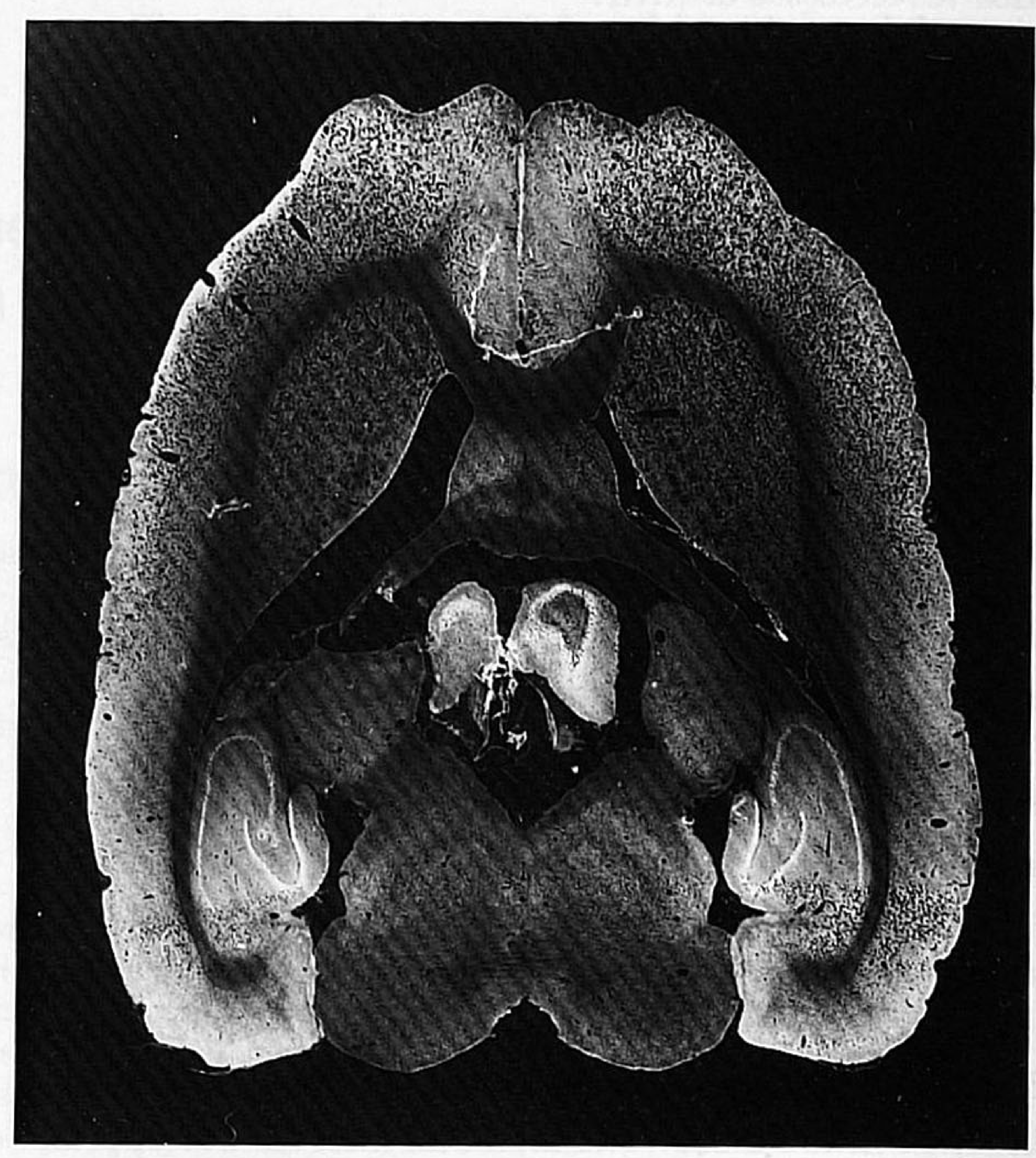

Fig. 3. Illustration of fornix lesion in Cresyl violet-stained horizontal section rat at level showing entire semi-circular curvature of the anterior commissure. Photograph made by projecting histologic section using an enlarger directly onto photographic paper.

from similar treatment groups were combined. The fimbria-fornix transection resulted in a significant reduction of AChE-positive cells in the ipsilateral medial septum $\left(F_{1,14}=31.651, P<0.0001\right)$. This effect was not attenuated by the nimodipine treatment.

The frequency scores of locomotion, hole-poke, rearing, and grooming in the open field did not differ among groups. The data are presented in Fig. 4.

There were no significant differences in the water maze latency for groups of lesioned animals treated with



Fig. 4. Mean frequencies of occurrence ( \pm S.E.M.) of open field behaviors in Expt. 2. LV, $n=10$; LN, $n=10$; SV, $n=9$, SN, $n$ $=9$. 
$0.5 \mathrm{mg} / \mathrm{kg}, 1.0 \mathrm{mg} / \mathrm{kg}, 5.0 \mathrm{mg} / \mathrm{kg}$, or vehicle. The data are comparable to those of the lesioned animals shown in Fig. 2. (Data not shown.)

This study demonstrates a strong learning deficit resulting from unilateral lesions of the fimbria-fornix as assessed by the use of this complex water maze. Differences between lesioned and sham-operated controls became apparent by the second day of testing. Accompanying the behavioral effects of the lesions was a loss of cholinergic innervation in the hippocampus and of acetylcholinesterase-stained cells in the medial septal area ipsilateral to the lesion.

Administration of the $\mathrm{Ca}^{2+}$ channel antagonist, nimodipine, at the doses and procedures employed in these experiments failed to attenuate any of the behavioral or histological effects of the lesion. The open field measures of locomotion, hole-poke, rearing, and grooming did not reveal any differences among groups of animals, suggesting that the dose of nimodipine used did not likely produce motoric abnormalities that would account for changes found in the water maze.

In the time allowed our animals for recovery, we found no indication of an increase in AChE staining in the hippocampus. It is possible that 28 days is insufficient for such an increase to occur but it is also possible that the completeness of our lesions would have prevented an increase being observed at any postoperative interval. Some authors assert that some residual sparing of

1 Armstrong, D.M., Terry, R.D., Deteresa, R.M., Bruce, G., Hersh, L.B. and Gage, F.H., Response of septal cholinergic neurons to axotomy, J. Comp. Neurol., 264 (1987) 421-436.

2 Blaker, S.N., Armstrong, D.M. and Gage, F.H., Cholinergic neurons within the rat hippocampus: response to fimbria-fornix transection, J. Comp. Neurol., 272 (1988) 127-138.

3 Danks, A.M., Oestreicher, A.B., Isaacson, R.L., Spruijt, B.M. and Gispen, W.H., Possible neurotrophic effects of Nimodipine on cognitive behavior and histochemical distribution of AChE and B-50 (GAP 43) in unilateral fimbria-fornix lesioned rats, Soc. Neurosci. Abstr., 14 (1988) 1246.

4 Dravid, A.R. and Van Deusen, E.B., Recovery of choline acetyltransferase and acetylcholinesterase activities in the ipsilateral hippocampus following unilateral, partial transection of the fimbria in rats, Brain Research, 277 (1983) 169-174.

5 Dunnett, S.B., Low, W.C., Iversen, S.D., Stenevi, U. and Björklund, A., Septal transplants restore maze learning in rats with fimbria-fornix lesions, Brain Research, 251 (1982) 335-348.

6 Gage, F.H., Björklund, A. and Stenevi, U., Reinnervation of the partially deafferented hippocampus by compensatory collateral sprouting from spared cholinergic and noradrenergic afferents, Brain Research, 268 (1983) 27-37.

7 Gage, F.H., Wictorin, K., Fischer, W., Williams, L.R., Varon, S. and Björklund, A., Retrograde cell changes in medial septum and diagonal band following fimbria-fornix transection: quantitative temporal analysis, Neuroscience, 19 (1986) 241-255.

8 Gasser, U.E., Van Deusen, E.B. and Dravid, A.R., Homologous cholinergic efferents spared by partial fimbrial lesions contribute to the recovery of hippocampal cholinergic enzymes in adult rats, Brain Research, 367 (1986) 368-373.

9 Hedreen, J.C., Bacon, S.J. and Price, D.L., A modified cholinergic fibers reaching from the septal area to the hippocampus is necessary for the return of cholinergic enzymes after interrupting the fornix ${ }^{8}$. Following complete fimbria-fornix transection, no recovery of $\mathrm{AChE}$ was observed even when the animals were allowed to recover for as long as a year ${ }^{4}$. However, others have reported increases in AChE in the most temporal aspects of the hippocampus, possibly indicating a sprouting of cholinergic fibers from piriform regions or via ansa lenticularis, after complete fimbria-fornix lesions ${ }^{5}$. Because of the possible importance of complete lesions for anatomial and/or behavioral recovery, great care must be used to evaluate whether or not lesion completeness is achieved. In the present experiment the authors feel confident that complete lesions of the fimbria-fornix were produced since two means of verification were employed with extremely strict criteria. Therefore, it is possible that because there may have been no residual remaining fibers, no recovery of function or cellular protection of septal cells was observed. Nimodipine may function to enhance and/or extend the potential of remaining fiber systems after damage, both central and peripheral. Without some remaining fibers, the drug may not be effective. Complete lesions of the fimbria-fornix may eliminate the transport of any trophic factors from the hippocampus to the septal region and nimodipine may act to enhance the effectiveness of these substances, but not act as an independent trophic influence, itself.

histochemical technique to visualize acetylcholinesterase-containing axons, J. Histochem. Cytochem., 33 (1985) 134-140.

$10 \mathrm{Hefti}$, F., Dravid, A. and Hartikka, J., Chronic intraventricular injections of nerve growth factor elevate hippocampal choline acetyltransferase activity in adult rats with partial septo-hippocampal lesions, Brain Research, 293 (1984) 305-311.

11 Isaacson, R.L., Shen, Y. and Mandel, A., Effects of nimodipine on medial septal area cells after section of the fimbria-fornix, Restor. Neurol. Neurosci., 2 (1990) 1-7.

12 Karnovsky, M.J. and Roots, L., A 'direct-coloring' thiocholine method for cholinesterases, J. Histochem. Cytochem., 12 (1964) 219-221.

13 Nyakas, C., Markel, E., Bohus, B., Schuurman, T. and Luiten, P.G.M., Protective effects of the calcium antagonist nimodipine on discrimination learning deficit and impaired retention behavior caused by prenatal nitrite exposure in rats, Behav. Brain Res., 38 (1990) 69-76.

14 Nyakas, C., Markel, E., Kramers, R.J.K., Gaspar, E., Bohus, B. and Luiten, P.G.M., Effects of nimodipine on hypoxiainduced learning and memory deficits. In J. Traber and W.H. Gispen (Eds.), Nimodipine and Central Nervous System Function: New Vistas, F.K. Schattauer, Stuttgart, 1989, pp. 175-194.

15 O'Brien, T.S., Svendsen, C.N., Isacson, O. and Sofroniew, M.V., Loss of True Blue labelling from the medial septum following transection of the fimbria-fornix: evidence for the death of cholinergic and non-cholinergic neurons, Brain $R e$ search, 508 (1990) 249-256.

16 Pitsikas, N., Spruijt, B., Algeri, S. and Gispen, W.H., The ACTH/MSH (4-9) analog ORG 2766 improves retrieval of information after a fimbria-fornix transection, Peptides, in press.

17 Reinstein, D.K., Hannigan, J.H. and Isaacson, R.L., Time 
course of certain behavioral changes after hippocampal damage and their alteration by dopaminergic intervention into nucleus accumbens, Pharmacol. Biochem. Behav., 17 (1982) 193-202.

18 Siesjö, B.K., Cell damage in the brain: a speculative synthesis, J. Cereb. Blood Flow Metab., 1 (1981) 155-185.

19 Siesjö, B.K. and Smith, M.L., Acidosis-related brain damage immediate and delayed events, mechanisms of cerebral hypoxia and stroke, Adv. Behav. Biol., 35 (1988) 57-72.
20 Spruijt, B., Pitsikas, N., Algeri, S. and Gispen, W.H., Org 2766 improves performance of rats with unilateral lesions in the fimbria fornix in a spatial learning task, Brain Research, 527 (1990) 192-197.

21 Van der Zee, C.E.E.M., Schuurman, T., Traber, J. and Gispen, W.H., Oral administration of nimodipine accelerates functional recovery following peripheral nerve damage in the rat, Neurosci. Lett., 83 (1987) 143-148. 